Jorge Martín Rodríguez Hernández ${ }^{1}$ Diana Patricia Rodríguez Rubiano ${ }^{2}$ Juan Carlos Corrales Barona ${ }^{3}$

${ }^{1}$ Pontificia Universidad Javeriana Bogotá. Carrera 7 no $^{\circ} 40-90 / 5^{\circ}$. Bogotá Colombia. jrodriguez.h@ javeriana.edu.co ${ }^{2}$ Escuela de Salud Pública de México.

${ }^{3}$ Escuela de Salud Pública, Universidad del Valle de Colombia.

\title{
Barreras de acceso administrativo a los servicios de salud en población Colombiana, 2013
}

\author{
Barriers to administrative access to health services \\ in the Colombian population, 2013
}

Abstract The scope of this paper is to characterize the main barriers faced by the Colombian population when they attempt to gain access to health services. It is an observational, descriptive and exploratory study using both quantitative and qualitative techniques. It was based on the records of Petitions, Complaints, Claims and Suggestions sent to the National Department of Health of Colombia between January 2102 and June 2013. In-depth interviews were conducted with users of health services, as well as officials of the Health Promoting Companies and Outlets for Right to Health of the Health Department of Bogotá. The study is based on domains proposed by Frenk et al for effective access to health services. Users of this study found limitations starting at admission and search for care due to communication failures in accessing health services. For ongoing care services, they encountered barriers resulting from the need for authorizations, lack of availability of specialist medical appointments, surgical procedures and drug delivery. Based on the findings, the conclusion reached is that the barriers perceived by users generate negative perceptions and harmful consequences both for them and for their families. Key words Barriers to Access, Health services, Health systems, Colombia
Resumen El artículo tiene como propósito caracterizar las principales barreras que sufre la población colombiana al intentar acceder a los servicios de salud. Es un observacional descriptivo exploratorio que emplea técnicas tanto cuantitativas como cualitativas; se basó en los registros de Peticiones, Quejas, Reclamos y Sugerencias que llegaron a la Superintendencia Nacional de Salud de Colombia entre enero-2102 y Junio-2013; además, empleó entrevistas a profundidad a usuarios de los servicios de salud, a funcionarios de las Empresas Promotoras de Salud de Bogotá y de Puntos por el Derecho a la Salud de la Secretaría de Salud de Bogotá. Los usuarios de este estudio evidenciaron limitaciones desde la entrada y búsqueda de la atención por fallas en la comunicación para acceder a los Servicios de Salud; en la continuidad del servicio, se encuentran con barreras derivadas por las autorizaciones, falta de oportunidad para citas médicas especializadas, procedimientos quirúrgicos y entrega de medicamentos. Con base a los hallazgos se puede concluir que las barreras que perciben los usuarios generan percepciones negativas y efectos perjudiciales tanto para éstos, como para sus familias.

Palabras clave Barreras de acceso, Servicios de salud, Sistemas de Salud, Colombia 


\section{Introducción}

Las barreras de acceso a los servicios de salud (SS) desde las Empresas Administradoras de Beneficios (EAPB), son el conjunto de estrategias técnico-administrativas que éstas interponen para negar, dilatar o no prestar este tipo de servicios a sus afiliados. En este estudio se observa que Peticiones, Quejas, Reclamos y Solicitudes (PQRS) siguen en aumento pese a la normatividad que existe en Colombia para controlarlas. Son múltiples las justificaciones que las EAPB interponen frente a las experiencias vividas por los usuarios del Sistema de Salud Colombiano (SSC) para acceder a los SS, que sumadas a las barreras geográficas, económicas y organizativas aumentan la inequidad en salud ${ }^{1,2}$. Se han identificado diversas barreras generadas por las Empresas Promotoras de Salud (EPS), un tipo de EAPB, al acceso a los Planes de Beneficios establecidos en el Plan Obligatorio de Salud (POS), las cuales infringen el derecho a la salud establecido por la Constitución Política Colombiana ${ }^{3}$.

Como lo han comentado varios autores, la salud desde hace varios años pasó a ser vista como una "oportunidad de negocio", se ha enfocado en aspectos principalmente financieros y bajo una óptica gerencial, conllevando a que el lucro se convierta en el foco principal, desplazando la obtención de resultados en salud ${ }^{4}$; de esta forma, los problemas asociados a la negación y accesibilidad de los SS, se emplean como mecanismos de contención de costos, desencadenando consecuencias funestas para el bienestar de muchas personas y sus familias ${ }^{5-8}$. Los problemas asociados al acceso y utilización de los SS generan situaciones que incrementan la inequidad sanitaria ${ }^{9}$.

Es cierto que la cobertura del régimen contributivo, como del subsidiado, dentro del Sistema General de Seguridad Social en Salud (SGSSS), se ha incrementado ${ }^{10}$, no obstante, también es innegable que las barreras a los SS, han generado que día a día se interpongan miles de acciones legales para garantizar el derecho a la salud ${ }^{3,11}$. Los sistemas de salud, tienen la obligación de ser asequibles, equitativos, sostenibles y de calidad; no obstante, existen evidencias que indican que están debilitados en sus principales funciones de promover, restablecer y mejorar la salud individual y colectiva ${ }^{12}$. Esto se ve reflejado en el descontento de las personas ante la incapacidad de éstos para satisfacer sus necesidades, con el agravante de incrementar los gastos de bolsillo ${ }^{13,14}$.

Donabedian $^{15}$ y Frenk ${ }^{16}$, adoptan la accesibilidad como un concepto referido al "grado de ajuste" entre las características de la población y los recursos de atención que disponen las Instituciones Prestadoras de Salud (IPS). Aday \& Andersen $^{17}$, equiparan este concepto con el uso de los SS. Restrepo y cols, plantean el acceso a los SS como el proceso mediante el cual puede lograrse atención y satisfacción ${ }^{18}$. Donabedian ${ }^{15}$ argumenta que "la prueba de acceso es uso de servicios, no solo la presencia de una instalación”. Por tanto, el acceso a los SS hace referencia al proceso por el cual las personas buscan y logran atención ${ }^{16,17}$. Frenk propone tres dominios para el acceso a los SS: 1) Dominio estrecho, comprende la búsqueda de atención. 2) Dominio intermedio, comprende consecución y continuación de la atención. 3) Dominio amplio, corresponde a todo el proceso, desde el deseo de atención hasta la satisfacción brindada por los SS.

Aday y Andersen, consideran tres dimensiones básicas para entender la relación entre los usuarios de servicios y el sistema de salud: la política, el acceso potencial y el acceso real ${ }^{17}$. La primera dimensión se refiere a las acciones planeadas y dirigidas por el Estado para mejorar el acceso a los SS, incluye la organización del sistema de prestación, la asignación de recursos y la definición de criterios de elegibilidad ${ }^{17}$. La segunda, aborda la interacción entre las características del sistema de salud, tiene en cuenta la disponibilidad y oferta de servicios, y las del individuo, donde influyen factores como recursos disponibles de las personas, seguros de salud, características socio-demográficas, estado de salud, localización y portafolio de servicios con que cuenta el proveedor, entre otras. La tercera, el acceso real, hace referencia al uso efectivo de los SS y a las experiencias de los usuarios frente a éste.

El incumplimiento de las acciones para acceder a los servicios de manera ágil y oportuna trae efectos al usuario a nivel familiar, laboral, económico, social, entre otros, inhabilitándolo no sólo a él, sino a su familia. Esta situación genera procesos de inequidad, como lo reportan varios investigadores $^{1,2,19}$. El acceso a los SS se ha convertido en una problemática constante, donde las leyes del mercado determinan la estructura y el funcionamiento de los mismos.

El panorama anterior indica que es necesario cumplir la legislación, tal como está planteado en la Ley 1122 de $2007^{20}$, que establece que dentro de las funciones de la salud pública está garantizar de manera integral la salud de la población por medio de acciones de salubridad tanto a nivel individual como colectivo. En este contexto, la Superintendencia Nacional de Salud de Colombia 
(SNS) es la entidad encargada de fortalecer mecanismos de monitoreo a las áreas de las EAPB (administrativa, técnica científica, económica, entre otras). Debe garantizar el cumplimiento de derechos de los usuarios en el SSC y hacer cumplir los deberes por parte de los diferentes actores del sistema.

Este estudio tuvo como objetivo caracterizar las principales barreras que sufre la población colombiana al intentar acceder a los servicios de salud.

\section{Metodología}

Diseño, población y fuentes de información

Esta investigación corresponde a un estudio descriptivo exploratorio que caracterizó las principales barreras que encuentran algunos usuarios al intentar acceder a los SS en Colombia. Es un estudio con enfoques cuantitativos y cualitativos. Las fuentes fueron 1. Registros de la SNS con base a PQRS que hacían por diferentes medios; 2. Análisis de casos con entrevistas realizadas a usuarios, funcionarios de una entidad distrital y EPS en el Distrito Capital de Bogotá. La información se recolectó entre julio y septiembre de 2013.

\section{Recolección y procesamiento de la información}

Los registros de la SNS, se revisaron, homogenizaron, depuraron y limpiaron; se usó excel y stata versión 11.0. Las variables analizadas para el período de estudio fueron mes, medio por el que se estableció las PRQS, entidad vigilada, sexo, edad, nivel educativo y etnia del solicitante, régimen de afiliación, motivo general y específico de las PQRS. De esta información se obtuvieron frecuencias absolutas y relativas.

Se diseñaron entrevistas semiestructuradas y en profundidad que tenían en cuenta los dominios para el acceso a los servicios de salud (estrecho, intermedio y amplio), indagando por los motivos que conllevaban a la negación, dilación o postergación de la atención de los SS. Cada dominio fue clasificado como barreras administrativas (Tipo I, II y III) para el acceso; para cada barrera se generaron atributos específicos, tal como se muestra en el Cuadro 1. El abordaje cualitativo uso el estudio de caso con el fin de obtener testimonios sobre las barreras que viven los usuarios al intentar acceder a los SS; se exploraron las repercusiones en el entorno personal, familiar, laboral, social y cultural de éstos. Se incluyeron usuarios que cumplieran con los siguiente criterios de inclusión: estar afiliados(as) a uno de los dos regímenes (contributivo o subsidiado), ambos sexos, mayores de 18 años y menores de 70 años, que hubieran manifestado una barrera en el último año. El reclutamiento de participantes se hizo por "bola de nieve", en la cual los afectados refirieron a otros usuarios, delimitando el número de casos por el criterio de saturación ${ }^{21}$.

Además, se seleccionaron funcionarios del área administrativa de las EPS, con más antecedentes de PQRS, para indagar sobre las estrategias que utilizaron para solucionar o retardar la atención en la prestación del SS a los usuarios. Al final de la entrevista, se solicitaron los diagnósticos y planes de mejoramiento donde se verificaran acciones correctivas para el acceso a los SS. También se entrevistó a funcionarios de los puntos por el derecho a la salud (PDS) pertenecientes a la Secretaría de Salud Distrital de Bogotá (SSD), dependencia encargada de promover los derechos en salud de los usuarios.

Las entrevistas se recogieron en grabaciones que luego fueron transcritas y analizadas por $s u$ contenido ${ }^{22}$ a través de códigos, categorías y subcategorías de todos los niveles para su posterior validación. Se empleó Atlas ti versión 7.0. El análisis se hizo teniendo en cuenta los dominios mencionados; se realizó para usuarios, funcionarios de EPS y de PDS.

\section{Triangulación e interpretación de la información}

Una vez obtenida la información, el análisis se desarrolló en dos fases 1 . Un proceso deductivo de codificación para asegurar que los datos empíricos estuvieran ampliamente explorados y fuera suficiente para producir conocimiento. 2 . Un proceso inductivo basado en el marco teórico propuesto y en la revisión de la literatura. Para asegurar la validez y el rigor metodológico, se generaron procesos de triangulación de métodos con información cuantitativa y cualitativa. Se generaron posibles explicaciones que sintetizaran los datos recabados sobre las barreras al acceso y oportunidad en la atención interpuestas por las EPS para la prestación de los SS y posibles efectos en la salud de los participantes.

\section{Aspectos éticos}

La presente investigación, es considerada sin riesgo; no realizó ninguna manipulación o mo- 
Cuadro 1. Resultados reportados por los diferentes actores en el proceso de acceso a los servicios de salud. Distrito Capital. 2013.

\begin{tabular}{|c|c|c|c|}
\hline $\begin{array}{l}\text { Tipo de } \\
\text { barrera }\end{array}$ & Usuarios & $\begin{array}{c}\text { Funcionarios } \\
\text { de EPS }\end{array}$ & $\begin{array}{c}\text { Funcionarios } \\
\text { de PDS }\end{array}$ \\
\hline $\begin{array}{l}\text { Tipo I } \\
\text { Demora en } \\
\text { la asignación } \\
\text { de citas } \\
\text { Caídas del } \\
\text { sistema } \\
\text { No registro } \\
\text { en bases de } \\
\text { datos }\end{array}$ & $\begin{array}{l}\text { Teléfonos ocupados o no } \\
\text { contestan. } \\
\text { Llamen cada hora para } \\
\text { lograr la cita } \\
\text { No aparece pago del servicio } \\
\text { Inseguridad de pedir cita } \\
\text { por ahí, por una experiencia } \\
\text { que tuve. } \\
\text { No hay agenda, sobre todo } \\
\text { con especialidades } \\
\text { Esté llamando o viniendo } \\
\text { para ver cuando hay agenda } \\
\text { Aparece desafiliado o } \\
\text { inactivo }\end{array}$ & $\begin{array}{l}\text { No tenemos un nivel de quejas } \\
\text { importante por esa variable } \\
\text { Contamos con Call Center en el } \\
\text { que deben contestar el } 80 \% \text { de las } \\
\text { llamadas antes de } 20 \text { segundos. } \\
\text { Tenemos una persona que se contacta } \\
\text { con la IPS. Para verificar si la planta } \\
\text { está dañada o si se cambió el número } \\
\text { telefónico. } \\
\text { Existe un canal telefónico propio, por } \\
\text { el cual hay intervención directa de la } \\
\text { organización, para responder a esas } \\
\text { llamadas. } \\
\text { La no atención del usuario es porque } \\
\text { paga el día que necesita del servicio, } \\
\text { por tanto no aparece en el sistema. }\end{array}$ & No reportan \\
\hline $\begin{array}{l}\text { Tipo II } \\
\text { Negaciones } \\
\text { del servicio } \\
\text { Dilación en } \\
\text { la atención } \\
\text { Trabas } \\
\text { burocráticas }\end{array}$ & $\begin{array}{l}\text { Ir a varios sitios para } \\
\text { autorizar, reclamar } \\
\text { medicamentos, toma de } \\
\text { exámenes y eso genera } \\
\text { gastos, que no hay muchas } \\
\text { veces } \\
\text { Pedir fotocopias y } \\
\text { resúmenes de historia } \\
\text { Demoras en la autorización } \\
\text { para que me operen de la } \\
\text { rodilla, siendo del POS } \\
\text { Esperar citas con el } \\
\text { especialista hasta meses } \\
\text { Falta uniformidad entre los } \\
\text { funcionarios para orientar } \\
\text { al usuario correctamente. } \\
\text { No he sido atendida por } \\
\text { inconsistencia en la base de } \\
\text { datos }\end{array}$ & $\begin{array}{l}\text { Por no tener la autorización del } \\
\text { servicio } \\
\text { Por tener órdenes vencidas } \\
\text { Falta de comunicación entre IPS e } \\
\text { IPS sobre la no prestación de ciertos } \\
\text { servicios } \\
\text { Letra ilegible de médicos, toca } \\
\text { corroborar información } \\
\text { Desconocimiento de la norma por las } \\
\text { IPS sobre lo que ha entrado de nuevo } \\
\text { en el POS retrasando la atención al } \\
\text { usuario }\end{array}$ & $\begin{array}{l}\text { No hay agenda especialmente } \\
\text { para especialidades } \\
\text { como medicina interna, } \\
\text { oftalmología, cirugía, } \\
\text { urología, demoras de } 2 \text { a } 3 \\
\text { meses } \\
\text { Problemas en la programación } \\
\text { de cirugías } \\
\text { Las autorizaciones son una } \\
\text { de la grandes barreras del } \\
\text { complejo administrativo que } \\
\text { no debe asumir el paciente } \\
\text { Le falta un dato a la fórmula } \\
\text { y no autorizan la entrega del } \\
\text { medicamento }\end{array}$ \\
\hline
\end{tabular}

dificación intencionada de variables y aspectos biológicos, fisiológicos, sicológicos o sociales en algún participante ${ }^{23}$; se eliminaron los datos de identificación para garantizar el anonimato y confidencialidad de los participantes. A los usuarios entrevistados, al igual que a los directivos de las EPS que participaron en la investigación, se les leyó un consentimiento informado aprobado por el Superintendente Delegado de Protección al Usuario y Participación Ciudadana donde se explicaban los objetivos, propósitos, fines del estudio y se garantizaba la confidencialidad por su participación en la investigación. Aquello(a)s usuario(a)s que aceptaron participar fueron contactados para fijar la fecha de la entrevista en un espacio que asegurase la confidencialidad.

\section{Resultados}

A continuación se hace una descripción de los principales resultados de las PQRS que hicie- 


\begin{tabular}{|c|c|c|c|}
\hline \multicolumn{4}{|c|}{ Cuadro 1. continuación } \\
\hline $\begin{array}{l}\text { Tipo de } \\
\text { barrera }\end{array}$ & Usuarios & $\begin{array}{c}\text { Funcionarios } \\
\text { de EPS }\end{array}$ & $\begin{array}{c}\text { Funcionarios } \\
\text { de PDS }\end{array}$ \\
\hline $\begin{array}{l}\text { Tipo III } \\
\text { Negación del } \\
\text { servicio } \\
\text { Dilación en la } \\
\text { atención } \\
\text { Insatisfacción } \\
\text { por barreras }\end{array}$ & $\begin{array}{l}\text { Yo puse AT y varios } \\
\text { desacatos, porque no me } \\
\text { querían atender y ese tumor } \\
\text { cada día estaba más grande } \\
\text { Tuve que esperar cuatro } \\
\text { meses para que me viera el } \\
\text { especialista } \\
\text { Pésimo el servicio, } \\
\text { estuvimos llamando al } \\
\text { área de referencia y contra } \\
\text { referencia y no fue posible, } \\
\text { y era urgente el traslado } \\
\text { Sigo luchando hasta lograr } \\
\text { lo que necesito } \\
\text { Busco la oficina de atención } \\
\text { al usuario }\end{array}$ & $\begin{array}{l}\text { Necesitamos verificar por medio de } \\
\text { la autorización, de acuerdo a la ley } \\
\text { si es POS o no POS } \\
\text { Se niegan procedimientos o } \\
\text { tratamientos No POS, cuando son } \\
\text { enviados por médicos particulares o } \\
\text { que no son prioritarios } \\
\text { Se niegan procedimientos que no } \\
\text { están aprobados por asociaciones } \\
\text { científicas internacionales y sin } \\
\text { soportes clínicos. } \\
\text { Los desacatos son porque los } \\
\text { usuarios quieren acceder a algo que } \\
\text { no tienen derecho, pero tienen el } \\
\text { respaldo del señor juez } \\
\text { Existen usuarios fraudulentos } \\
\text { y dañinos, que por medio de la } \\
\text { tutela logran lo que quieren y luego } \\
\text { venden lo solicitado } \\
\text { Las AT son impuestas por usuarios } \\
\text { de forma caprichosa } \\
\text { La forma más fácil de desangrar el } \\
\text { sistema, son por el tipo de tutelas } \\
\text { que suelen poner }\end{array}$ & $\begin{array}{l}\text { Hay trabas y desorden en las } \\
\text { EPS } \\
\text { Hay casos de pacientes con } \\
\text { cáncer que han llegado a los } \\
\text { PDS donde a través de nuestra } \\
\text { intervención se han salvado } \\
\text { sus vidas } \\
\text { Se acude a colaborarles en } \\
\text { interponer la AT que se } \\
\text { le agrega lo más grave, ,el } \\
\text { desacato } \\
\text { La gente llega llorando y con } \\
\text { toda la razón y diciendo nadie } \\
\text { resuelve nada. } \\
\text { El sistema es muy agresivo } \\
\text { con la gente de la tercera } \\
\text { edad, discapacitados y con } \\
\text { enfermedades de alto costo, a } \\
\text { quienes nadie las atiende... } \\
\text { La gente entutela por cosas } \\
\text { que tienen derecho }\end{array}$ \\
\hline
\end{tabular}

ron los usuarios por diferentes medios a la SNS: telefónico $(62 \%)$, electrónico $(25,5 \%)$, escrito $(8,3 \%)$ y chat $(1,1 \%)$. Para el período de estudio hubo 802.690 PQRS (Figura 1).

En la Figura 1 se observa que de las PQRS: $54,9 \%$ correspondió a información solicitada al Call Center (solicitud de orientación e información por los usuarios); 10,6\% correspondió a otros motivos (no reconocimiento de prestaciones económicas, falta de disponibilidad o inapropiado manejo del recurso humano y físico para la atención, solicitud de otra información a la SNS, entre otros); $34,5 \%$ correspondió a problemas relacionados con restricciones al acceso a los SS, motivo de la presente investigación.

En el Gráfico 1 se aprecia un incremento de PQRS, con promedio de 15.500 PQRS/mes, para el período de estudio. No fue posible analizar el comportamiento por edad, debido a que cerca de $47 \%$ de PQRS no registró este dato o había registros diversos. El sexo de los participantes tuvo mejor registro en 2012, 65,1\% de las PQRS fueron interpuestas por hombres y $33,4 \%$ por mujeres; para 2013, esta tendencia cambió, 50,9\% fueron interpuestas por mujeres y 47,5\% por hombres. En cuanto a la seguridad social, la mayoría refirió pertenecer al régimen contributivo (73\%), seguido del régimen subsidiado (19,5\%) y otros (Empresas de Medicina Prepagada, entidades adaptadas al sistema: 7,5\%).

Aunque para 2013 se reportó nivel educativo de quienes interponían PQRS, 58,4\% no tenía esta información específica (espacio vacío). Además, entre quienes tenían "algún registro", 55\% aparecía ninguno, 24\% secundaria y 15\% primaria. Algo parecido pasó con etnia, $60 \%$ no tiene información y $39 \%$ estaba como "No aplica".

De las PQRS derivadas de Restricciones al Acceso a Servicios de Salud, 52,5\%, fue por falta oportuna de atención; 20,1\% por demoras en autorizaciones; $11,9 \%$ por fallas en afiliación; $6,4 \%$ por restricciones económicas; $4,1 \%$ por negación de autorizaciones y 3,2\% por negaciones de la prestación de servicios, insumos o entrega de medicamentos: tanto POS, como No POS. (Que No corresponden al Plan Obligatorio de Beneficios en Salud existente en Colombia). De las PQRS derivadas por falta de atención oportuna, 51,7\%, fue 


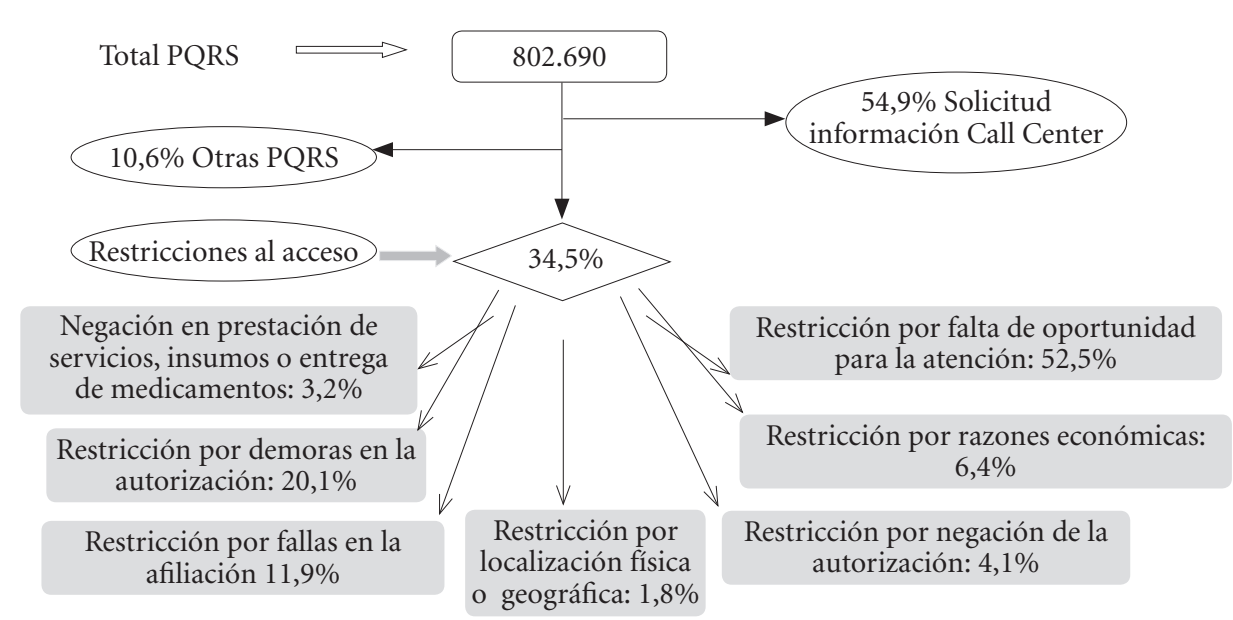

Figura 1. Algoritmo con PQRS realizadas a la Superintendencia Nacional de Salud, enero 2012-junio 2013.

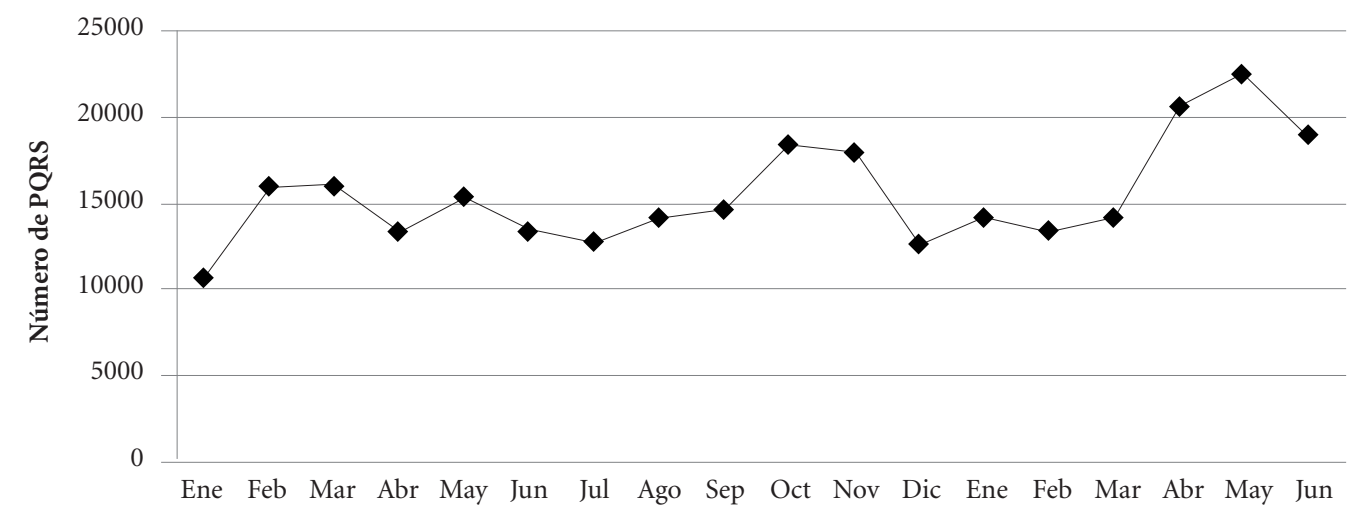

Gráfico 1. Distribución mensual de PQRS derivadas de restricciones al acceso a los servicios de salud 2012 2013.

por falta de oportunidad a citas especializadas (cardiología, ginecología, pediatría, entre otras), $10 \%$ por no oportunidad de servicios de promoción y prevención; $8,6 \%$ por no autorización de cirugías; por no autorización de medicación No POS (5\%) y medicación POS (4,7\%). El 20\% restante es por falta de oportunidad en la respuesta del Comité Técnico Científico. Aunque la Ley 100 se implementó hace dos décadas bajo los principios de solidaridad, eficiencia, eficacia, oportunidad, cobertura, el 6,4\% de las PQRS fue por restricciones económicas o capacidad de pago y
$1,8 \%$ por restricciones derivadas de la localización geográfica.

\section{Resultados cualitativos}

\section{Resultados con usuarios}

Se seleccionaron diez usuarios/as, nueve de los cuales se entrevistaron directamente y uno se analizó indirectamente ${ }^{24}$. Se incluyeron a usua- 
rios de los SS que cumplieran los criterios de inclusión planteados en el diseño del estudio; dos participantes iniciales tenían vínculo social-laboral con uno de los investigadores, quienes, a través de la técnica de "bola de nieve" sirvieron de base para incluir a otros participantes.

Las primeras limitaciones que reportaron los usuarios fueron las autorizaciones, múltiples desplazamientos para obtener citas de consulta médica general o especializada, epicrisis exigida para la autorización; tiempos largos de espera, congestión en los SS y remisiones a IPS sin convenio con la EPS. Además, si el especialista tratante remitía a otro especialista, conseguir esa nueva cita era complicado para la mayoría de usuarios: no hay agenda o tiene que verlo nuevamente el médico general.

Ante estas dificultades, varios usuarios mencionaron que recurrían a métodos paliativos, farmacias o médico cercano. Si contaban con recursos económicos, pagaban médico particular, y/o acudían a instancias legales como Acción de Tutela (AT). Algunas personas refirieron: Con Derechos de Petición o AT es que atienden rápido, Aun con la AT se violan derechos. Hubo un caso de desistimiento: Desisto de la atención, para evitar tanto obstáculo y permisos en el trabajo, a pesar de sentirme enferma. En el Cuadro 1 se encuentra la síntesis reportada por los usuarios ante las barreras percibidas.

También manifestaron diversas opiniones respecto a la gestión realizada por las instituciones encargadas de la protección al usuario: falta promover y capacitar en derechos y deberes, comunicar por diferentes medios la normatividad que beneficia al usuario al momento de encontrar barreras. Caracterizaron a la SNS como entidad poco resolutiva ante la problemática que vive el país; consideran que para mejorar las acciones de Inspección Vigilancia y Control (IVC), es necesario abrir puntos de atención personalizada en varias partes del país; supervisar y sancionar a EPS ante actos reiterados de incumplimiento.

\section{Resultados con funcionarios de EPS}

Se entrevistaron a 19 funcionarios, quienes conceptualizaron que las barreras de acceso corresponden a restricciones administrativas y/o de oportunidad, que los usuarios deben superar para acceder a los SS a los que tienen derecho; están relacionadas con aspectos geográficos y factores externos que no competen a las EPS, como el modelo económico, falta de normatividad, débil control de precios a medicamentos, poca oferta de especialistas, ausencia de sistemas de información unificados (SIU), entre otras. Reconocen que las quejas más frecuentes son inoportunidad de citas con especialistas; reconocimiento de incapacidades y licencias de maternidad; ajustes a cuotas moderadoras; demoras con autorizaciones; fallas en la comunicación con IPS; problemas de referencia y contra-referencia; inconformidad con el acto médico. En el Cuadro 1 se encuentran las síntesis reportadas por estos funcionarios.

Respecto a la atención en medicina general y especializada, mencionaron que para la primera no existen inconvenientes, la oportunidad está abajo de tres días. Para medicina especializada, reportaron inconvenientes por la poca oferta existente en el país, especialmente en neurología, fisiatría, dermatología, endocrinología, ortopedia, medicina interna, y nefrología. Para mejorar la oportunidad, funcionarios de una EPS, reportaron que cubren estos servicios con otros profesionales: reemplazan al médico internista por médico familiar; ortopedista para el manejo de Displasia de Cadera por pediatra; fisiatras por fisioterapia y oftalmólogos por optómetras. Varias EPS desconocían la oportunidad de citas para servicios como nutrición, psicología, trabajo social, terapias e imagenología.

Manifestaron que a los usuarios no les niegan servicios, siempre y cuando sean pertinentes; y tienen que gestionar pocas autorizaciones para niveles I y II. Para usuarios con cáncer, refirieron que no deben realizar ningún trámite, excepto acudir a la farmacia para reclamar el medicamento; son pacientes prioritarios para todas las EPS, donde es más benéfico acortar el tiempo de autorización. Estas afirmaciones, como se anotó antes y se verá adelante, no coinciden con lo reportado por los usuarios y funcionarios de los PDS.

En cuanto a la interposición de AT, reportaron que éstas deben usarse como último recurso y cuando se detecte que se ha vulnerado el derecho de las personas, se volvió algo cultural. Muchas AT son impertinentes. Los desacatos ocurren porque los usuarios quieren acceder a algo que no tienen derecho, pero tienen el respaldo del señor juez. Reconocen que existen efectos para usuarios y sus familias ante las barreras de acceso: puede llevar a la muerte, a consecuencias psicológicas y económicas por los desplazamientos para obtener el servicio.

Todos los funcionarios de las EPS, reportaron que tenían planes complementarios o medicina prepagada para acceder a los SS, comentaron: la inequidad en Colombia no existe, lo que pasa es que la medicina prepagada, la accede el usua- 
rio que puede pagar de su propio bolsillo, se tiene oportunidad a nuevas tecnologías.

Reportaron falta de acompañamiento de las entidades responsables de la normatividad del SGSSS, fallas de comunicación y débil articulación con la SNS; poca instrucción a los usuarios sobre deberes y forma correcta de usar los SS; dicen que es indispensable unificar tarifas para especialidades y verificar recurso humano para cubrir estas necesidades.

\section{Resultados con funcionarios de los PDS}

Se entrevistaron dos funcionarios, quienes reportaron que las barreras de acceso a los SS, son producto del modelo de salud Colombiano, a las que se suman limitantes geográficas. Los PDS nacen en respuesta a una necesidad sentida de la población vulnerable (pobre no asegurada y del régimen subsidiado), ante las dificultades encontradas al momento de requerir SS; realizan procesos de detección, revisión, análisis, orientación del caso para dar respuestas a los usuarios y ayudarlos a acceder al SS, incluyendo el empleo de acciones legales.

La SSD dispone de software donde registra y clasifica las PQRS; además de un sistema de identificación de barreras, implementado en IPS Públicas, EPS subsidiadas y PDS, donde revisan calidad, oportunidad, accesibilidad y continuidad de uso en los SS para generar acciones correctivas. Reportan que los afiliados al régimen contributivo, viven dicha problemática con el agravante que asumen copagos, sin embargo en ocasiones, no son atendidos oportunamente, generando gastos de bolsillo para suplir esta necesidad, llámese tratamiento, medicamento o cirugía. El nivel I es la puerta de entrada, de ahí en adelante comienza el martirio, con las múltiples barreras de acceso. En el Cuadro 1 se encuentra lo reportado por estos funcionarios en el proceso de atención a los usuarios.

Reportan que las EPS e IPS tienen como excusa, la no disponibilidad de una infraestructura tecnológica y no contar con funcionaros y evitar que a los usuarios les carguen los trámites entre EPS e IPS; eso vale plata y no les interesa la gente.

Los PDS han apoyado en la sensibilización frente a las barreras de acceso, para que los usuarios conozcan que sus derechos han sido vulnerados, que existen mecanismos de defensa, en otras instancias, las quejas se quedan en el trámite. Han capacitado a funcionarios de alcaldías locales y de juntas de acción comunal, para que exijan derechos y se empoderen; tienen planeado abrir un centro de producción de conocimiento. Refieren que la SNS necesita tener más presencia en el territorio, es un actor clave, para resolver las barreras de acceso. Existen casos donde no hay resolutividad por la SNS y los PDS tienen que gestionar para solucionar el problema. Recomiendan generar alianzas para trabajar de manera conjunta y hacer una atención más directa a la población.

\section{Discusión}

Esta investigación, dirigida a usuarios, funcionarios de EPS y PDS, confirma que las barreras de acceso a los SS en Colombia son múltiples. Los usuarios se enfrentan a limitaciones desde la entrada y búsqueda de la atención por fallas en la comunicación con las EPS e IPS para acceder a los SS; en la continuidad del servicio, se encuentran con barreras derivadas por las autorizaciones, falta de oportunidad para citas médicas especializadas, procedimientos quirúrgicos y entrega de medicamentos, lo que ocasiona percepciones negativas y efectos perjudiciales tanto para éstos, como para sus familias. Las barreras de acceso son generadas principalmente por el diseño del SGSSS y prácticas indebidas de algunas EPS, lo cual concuerda con lo reportado por otros autores ${ }^{7}$. Varios funcionarios de éstas, consideran que el acceso a los SS, se relaciona con el nivel adquisitivo, lo cual no coincide con los principios de equidad en salud y con la realidad social de la mayoría de la población Colombiana ${ }^{5,13}$.

Con los registros de la SNS y de la SSD se observó que las restricciones al acceso a los SS, han aumentado, afectando principalmente a personas de bajos recursos económicos ${ }^{25}$. Según los funcionarios de los PDS, existen múltiples causas por las que los usuarios del SGSSS interponen una AT; una de las principales son las autorizaciones; respecto a este punto, el Viceministro de Salud (2013) refirió que "el sistema no debe seguir moviéndose a través de la tutela, debe fluir con procesos administrativos eficaces para que la población acceda a los servicios de salud"26.

Aunque los usuarios recurren a la AT, en muchas ocasiones es desacatada, situación que pone en riesgo la vida. En el presente estudio se reflejó en la muerte de un menor ${ }^{24}$. La AT ha dejado de ser una disposición eficiente para la solución de las necesidades de salud, aunque sigue siendo usada como una medida para superar las barreras de acceso a los SS. Estos hallazgos, concuerdan con Múnera, quién refiere que las quejas son construcciones hechas por los usuarios sobre expe- 
riencias de atención en salud vividas y expresadas como insatisfactorias; donde la forma para romper las barreras de acceso es buscar ayuda por medios legales ${ }^{27}$. Vélez-Arango et al. ${ }^{11}$, encuentran expresiones similares a las relatadas tanto por los usuarios como por los funcionarios de los PDS frente al uso de las $\mathrm{AT}^{11}$. Las razones por las cuales los usuarios entablan AT y las justificaciones que mencionan los funcionarios de las EPS, para desacatarlas, concuerda con Yepes, quien los llama "encuentros dispares"28.

El Viceministro de Salud en 2013 consideraba que la ganancia que muestra Colombia en equidad es mucho mayor, en acceso a los SS, (medicamentos y servicios)..., situación refutada por Avellaneda-Trazona et al. ${ }^{26}$, quien afirma que las inequidades en el mercado de la salud continúan, al estar estratificadas con medicina para personas con mayor posibilidad de pago (medicina prepagada y/o servicios complementarios plus); medicina para sectores medios (régimen contributivo) y medicina para pobres (régimen subsidiado).... Hernández, en años previos, afirmaba que el sistema estaba montado sobre supuestos que no han cumplido con el aumento del empleo formal y crecimiento económico, con consecuencias para las clases sociales desfavorecidas ${ }^{6}$. Contrario ocurre en países como Inglaterra, Canadá e Irlanda donde las clases sociales pobres tienen mayor posibilidad de acceder a los SS, demostrando procesos de equidad ${ }^{1,2,29}$.

Así mismo, se identificaron percepciones de insatisfacción por parte de los usuarios (dominio amplio): déficit en la orientación, irrespeto de funcionarios y falta en la continuidad de tratamientos. Situaciones de malestar similar se encontraron en estudios realizados en México, quienes reportaron mala calidad en la atención, insatisfacción relacionada con negligencia y maltrato por parte del médico ${ }^{30,31}$. Algo similar se ha observado en Chile, Brasil y en países Centro-americanos, con incremento de demandas, reclamos, y exclusiones del Sistema de Salud, reflejado en obstáculos para acceder a los servicios de salud en población general, en adultos mayores, mujeres, en poblaciones vulnerables, en especial a medicamentos y citas médicas especializadas ${ }^{9,32-35}$.

No se encontraron en la literatura estudios que revelen dificultades en la comunicación vía telefónica, siendo éste uno de los medios más empleados en la búsqueda de SS en Colombia. El principal motivo por el cual los usuarios reportaron utilizar los SS, fue la extrema urgencia, similar a lo hallado por Jaramillo et al. ${ }^{36}$, quien también reporta que el factor económico (cuotas moderadoras y copagos) además de moderar el uso de los SS, podría restringir el acceso a éstos, situación que fue declarada al menos por uno de los usuarios del presente estudio.

Se observó desconocimiento de la normatividad por parte de los usuarios, lo que ocasionó que fueran manipulados. Para disminuir esta brecha, Vélez reporta la existencia de instrumentos de política pública que orientan la garantía del derecho fundamental a la salud ${ }^{11}$. Algunas de estas herramientas son la Ley 1122/2007 (crea el defensor del usuario); Decreto 4747/2007, en sus Artículos 13 y 14 (prohíbe trasladar a los usuarios los trámites burocráticos para autorizaciones ante las EPS); Ley 1438/2011 (prohíbe el cobro de multas para citas médicas); la Resolución 1552 (establece asignación de citas en menos de tres días hábiles); Circular externa 056 (define los tiempos de espera para citas médicas y/o especializadas); Circular 005/2012 (garantiza el acceso oportuno e integral, en todos los niveles, a la mujer durante la gestación, parto o puerperio); Norma 1604/2013 (establece la entrega de medicamentos en menos de 48 horas en el domicilio del usuario); entre otras.

Las justificaciones dadas por los funcionarios de las EPS a las barreras de acceso, giraron alrededor de aspectos estructurales del SS, del modelo económico y de barreras geográficas, similar a lo reportado por otros investigadores ${ }^{37}$. Aunque los funcionarios de las EPS mostraron planes de mejoramiento para disminuir las barreras, varios no coincidieron con las PQRS existentes para cada una de ellas. Luego de una minuciosa revisión documental sobre el reemplazo de especialistas, para aumentar la oportunidad (médico internista por médico familiar; oftalmología por optometría), se encontraron diferencias en competencias y destrezas entre las especialidades originales y las sustitutas ${ }^{38-41}$.

Tal como se ha observado en otros escenarios $^{42}$, las barreras de acceso, han conllevado a efectos negativos en aspectos personales, emocionales, familiares, sociales, laborales y culturales; el estrés personal, emocional y familiar, son de las situaciones más frecuentes, al igual que las dificultades laborales por permisos reiterados. En el presente estudio, se observó la muerte de un menor por desacato de una tutela y no autorización de un procedimiento especializado. ${ }^{24}$

El estudio tiene algunas limitantes, dentro de las cuales es importante señalar: varios PQRS tenían datos heterogéneos y poco precisos sobre variables como edad, escolaridad y etnia, que limitó la caracterización sociodemográfica de las 
personas que los interponían; el volumen y la selección de los entrevistados quizá pudo ser limitado, no obstante, se considera que se cumplió con el principio de saturación teórica con este grupo de participantes; este trabajo no representa a la población Colombiana, sin embargo, se suma al cuerpo de evidencias sobre las barreras que viven los usuarios al intentar acceder a los servicios de salud.

El modelo de aseguramiento que existe en Colombia ${ }^{43}$, establece que es necesario brindar a la población acceso a los SS, bajo los principios de universalidad, solidaridad y equidad; no obstante, pese a que los usuarios tienen la posibilidad de estar afiliados al régimen subsidiado o contributivo, el acceso real ante un problema de salud, no concuerda con el acceso potencial, para lo cual se deben implementar y hacer cumplir los mecanismos de política pública existentes en la legislación para mitigar esta problemática.

\section{Colaboradores}

JMR trabajó en la concepción, diseño del estudio, en el análisis de los resultados al igual que en la escritura del manuscrito y aprobación de la versión final. DPR en el diseño del estudio, en la recolección y análisis de la información, en la redacción y aprobación de la versión final. JCC trabajó en la concepción, diseño del estudio, en el análisis de los resultados y aprobación de la versión final.

\section{Agradecimientos}

$\mathrm{Al}$ área delegada de protección al usuario y participación ciudadana de la Super Intendencia Nacional de Salud por haber brindado la oportunidad de realizar este estudio; a los usuarios anónimos, los cuales de forma desinteresada participaron en la presente investigación. Al profesor Francisco José Yepes Lujan, director de Postgrados en Administración en Salud de la Pontificia Universidad Javeriana, por su lectura crítica al presente documento. 


\section{Referencias}

1. Braveman P, Gruskin S. Defining equity in health. J Epidemiol Community Health 2003; 57(4):254-258.

2. Whitehead $\mathbf{M}$. The concept and principles of equity and health. Geneva: Organización Mundial de la Salud; 2000.

3. Constitución Política de Colombia 1991. Capítulo 2. Derechos sociales, económicos y culturales. Articulo 48 y 49. Bogotá; 2012.

4. Franco-Agudelo S. Entre los negocios y los derechos. Rev Cub Salud Publica 2013; 39(2)268-284.

5. Abadia C, Oviedo D. Burocratic Itineraries in Colombia. A theoretical and methodological tools to access managed-care health care system. Soc Sci Med 2009; (68):1153-1160.

6. Hernández-Álvarez M. Derecho a la Salud en Colombia: Obstáculos Estructurales para su realización. Rev Salud Pública 2000; 2(2)121-144.

7. Agudelo CA, Cardona J, Ortega J, Robledo R, Sistema de salud en Colombia: 20 años de logros y problemas. Cien Saude Colet 2011; 16(6):2817-2828.

8. Tobón SA. Relatoría del foro "¿Para dónde va el sistema de salud en Colombia?” Un análisis al sistema de salud en Colombia. Revista Soluciones de Postgrado EIA 2009; 4:7-18.

9. Acuña C, Marin N, Mendoza A, Emmerick ICM, Luiza VL, Azeredo TB. Determinantes sociales de la exclusión a los servicios de salud y a medicamentos en tres países de América Central. Rev Panam Salud Pública 2014; 35(2):128-135.

10. Ministerio de Salud y Protección Social de Colombia. Situación actual de las EPS de los regímenes contributivo y subsidiado en Colombia. [consultado 2013 jun 1]. Disponible en http://www.minsalud.gov.co/Documentos\%20y\%20Publicaciones/Situaci\%C3\%B3n\%20 actual $\% 20 \mathrm{de} \% 20 \mathrm{las} \% 20 \mathrm{EPS} \% 20 \mathrm{de} \% 20 \mathrm{los} \% 20 \mathrm{reg} \%$ C3\%ADmenes\%20contributivo\%20y\%20subsidiado\%20en $\% 20$ Colombia.pdf

11. Vélez-Arango A, Realpe-Delgado C, Gonzaga-Valencia J, Castro-Castro AP. Acción de Tutela, Acceso y Protección del Derecho a la Salud en Manizales. Rev. Salud Pública 2007; 9 (2):297-307.

12. Organización Mundial de la Salud (OMS). Discapacidad y salud. [consultado 2013 mayo 1]. Disponible en: http://www.who.int/mediacentre/factsheets/fs352/es/

13. Rodríguez-Acosta S. Barreras y determinantes del acceso a los servicios de salud en Colombia [tesis]. Barcelona: Universidad Autónoma de Barcelona; 2010.

14. Mezquita M, Pavlicich V, Benitez S. Percepción de la calidad de atención y accesibilidad de los consultorios pediátricos públicos en Asunción y Gran Asunción. Rev Pediatr 2008; 35(1):11-17.

15. Donabedian A. Models for Organizing the Delivery of Health Services and Criteria for Evaluating them. The Milbank Memorial Fund Quarterly 1972; 50(4):103154

16. Frenk J. El concepto y la medición de la accesibilidad. Salud Pública Mex 1985; 27(5):438-453.

17. Aday L, Andersen R. A framework for the study of access to medical care. Health Services Research 1974; 9(3):208-220.

18. Restrepo J, Echeverri E, Vásquez J, Rodríguez S. El seguro subsidiado y el acceso a los servicios de salud. Teoría, contexto colombiano y experiencia en Antioquia. Medellín: Centro de Investigaciones Económicas; 2006.
19. Mejía-Mejía A, Sánchez-Gandur AF, Tamayo-Ramírez JC. Equidad en el acceso a servicios de salud en Antioquia, Colombia. Rev Salud Pública 2007;9(1):26-38.

20. Colombia. Gobierno Nacional. Ley 1122 de 2007. Por la cual se hacen algunas modificaciones al Sistema General de Seguridad Social en salud y se dictan otras disposiciones. Bogotá. 2008.

21. De Souza-Minayo MC. El desafío del conocimiento. Investigación cualitativa en salud. Buenos Aires: Lugar Editorial; 1997.

22. López-Aranguren E. Análisis de contenido. En García-Ferrando M, Ibáñez J, Alvira F. El análisis de la realidad social. Métodos y técnicas de investigación. Madrid: Editorial Alianza; 1994. p. 461-492.

23. Colombia. Ministerio de Salud (MS). Resolución 8430, por la cual se establecen las normas científicas, técnicas y administrativas para la investigación en salud. Bogotá: MS; 1993.

24. La enfermedad terminal del Sistema General de Seguridad Social en Salud. Bogotá: Radio Cadena Nacional (RCN); 2013.

25. Secretaria de Salud Distrital de Bogotá. Puntos por el Derecho a la Salud (PDS). Presentación de diapositivas. Bogotá: Secretaria de Salud Distrital de Bogotá; 2013.

26. Avellaneda-Trazona LC, Hernández-Álvarez M, RuizGómez F. Debate UN Alternativas para la reforma a la salud [Archivo de video]. [consultado 2013 jun 1]. Disponible en https://www.youtube.com/results?search_query

27. Múnera-Gaviria HA. La calidad de la atención en salud, más allá de la mirada técnica y normativa. Invest Educ Enferm 2011; 29(1):76-86.

28. Yépes C. Encuentros dispares generadores de dependencia en la atención en salud: estudio cualitativo sobre la percepción de la calidad en salud. Rev Fac Nac Sal Pública 2002; 20(1):9-22.

29. Mendoza-Sassi R, Béria JH. Health services utilization: a systematic review of related factors Cad Saude Publica 2001; 17(4):819-832.

30. Puentes-Rosas E, Gómez Dantés O, Garrido Latorre F. Trato a los usuarios en los servicios públicos de salud en México. Rev Panam Salud Publica 2006; 19(6):394402.

31. Ortiz-Espinoza RM, Muñoz-Juárez S, Torres-Carreño E. Satisfacción de los usuarios de 15 hospitales de hidalgo, México. Rev Esp Salud Pública 2004; 78(4):527537.

32. Reveiz L, Chapman E, Torres R, Fitzgerald JF, Mendoza A, Bolis M, Salgado O. Litigios por derecho a la salud en tres países de América Latina: revisión sistemática de la literatura. Rev Panam Salud Publica 2013; 33(3):213222.

33. Montoya-Aguilar C. Informe de la comisión presidencial de salud, diciembre 2010. Primeras reflexiones para el debate. Cuad Méd Soc Chile 2010; 50(4):340-343

34. Arredondo C, García RC, Palacios VA. ISAPRES, su regulación legal y la discriminación por riesgos en la cobertura de salud. Santiago: Universidad de Chile; 2012.

35. Da Cruz-Gouveia A, Morais-Duarte G, Gouveia-Figueiredo K, Oliveira-Duarte P, Sousa-Macau B. Acessibilidade aos serviços básicos de saúde: um caminho ainda a percorrer. Cien Saude Colet 2012; 17(11):29032912 
36. Jaramillo I, Martínez R, Olaya S, Reynales J, Uribe C, Castaño RA, Garzón E, Almeida C, Travassos C, Basto F, Angarita J, Céspedes JE. Efectos de la Reforma de la Seguridad Social en Salud en Colombia sobre la Equidad en el Acceso y la Utilización de los Servicios de Salud. Rev Salud Pública 2000; 2(2):145-164.

37. Travassos C, Oliveira EXG, Viacava F. Desigualdades geográficas e sociais no acesso aos serviços de saúde no Brasil: 1998 e 2003. Cien Saude Colet 2006; 11(4):975986.

38. Hudon C, St-Cyr Tribble D, Bravo G, Hogg W, Lambert M, Poitras ME. Family physician enabling attitudes: a qualitative study of patient perceptions. BMC Fam Pract 2013; 14:8.

39. Sarassa-Velásquez C, Carvajal-Casas J, Pérez-Nielsen C, Vélez-Cadavid A. Guía de Displasia de cadera en desarrollo. México: Asociación Col. de Facultades de Medicina; 2011. [confirmar las alteraciones en esta referencia]

40. Amarís P, Arenas E, Belalcár S, Carvajal J, Delgado MF, Diaz JF, Donado B, Escaf LJ, Gómez HF, Guzmán AM Mejía S, Melo LM, Molina M, Montoya G, Ocampo HH, Peña FY, Prada R, Francisco AQFJ, Rodríguez RI, Serrano JC, Serrano C, Vergara O, Zuluaga CU. Guías de Práctica Clínica. Bogotá: Sociedad Colombiana de Oftalmología; 2011.

41. Decker F. Physiatry vs. Physical Therapists. Disponible en http://work.chron.com/physiatry-vs-physical-therapists-20460.html. Consultado en septiembre 2013.

42. Nigenda G, Caballero M, González-Robledo LM. Barreras de acceso al diagnóstico temprano del cáncer de mama en el Distrito Federal y en Oaxaca. Salud Publica Mex 2009; 51(Supl. 2):S254-S262.

43. Vargas-Lorenzo I, Vázquez-Navarrete I, Mogollón-Pérez AS. Acceso a la atención en salud en Colombia. Rev Salud Pública 2010; 12(5):701-712.

Artigo submetido em 19/08/2014

Aprovado em 05/11/2014

Versão final apresentada em 07/11/2014 